\title{
New Polymers-New Uses
}

\author{
H. F. Mark*
}

\section{Introduction}

There are many indications that the conventional, classical fields for the application of organic polymers are gradually getting amply supplied with numerous materials which compete with each other and do not offer a very promising outlook for substantial further expansion. These fields are essentially the industries of textiles, packaging and printing materials, rubbers, plastics, coatings and adhesives which together represent a very large volume of synthetic and natural polymer consumption - in 1966 for the USA alone more than 120 billion lbs. or 60 million tons. The predictable saturation of these markets poses the question whether there exist additional large areas of industrial activity which could absorb substantial quantities of organic polymers if the properties and costs were so adjusted that their systematic and large scale application would present a technical and economic advantage for these industries.

It appears that the construction of cars, boats, airplanes and of buildings such as small and large homes, offices, hotels, factories and laboratories offers a promising chance for the consumption of considerable quantities of all kind of organic polymers if certain properties or, even better, certain combinations of properties could be conveniently built into these materials.

In order to get a rough estimate of the dimensions which are involved it should be mentioned that the car, boat, plane, house and home building activities in the USA in 1966 amounted to a total investment of about 150 billion dollars of which about $20 \%$ were spent for construction materials such as metals, concrete, brick, glass and wood. If

$*$ polymers are to be used in these areas they can serve as structural (load bearing) units or as finishing materials which service the system-car, boat, plane or building-with water, air, gas, fuel and electricity and provide for the finishing of its surface, of floors, ceilings and walls. Until now organic polymers have been mainly used in the finishing sector as floor tiles, coatings, covers, carpets, electrical and thermal insulating materials, window and door frames and as siding and roofing.

It is obvious that a successful expansion of polymer application in this field cannot be brought about by the development of one single propertyfor example, tensile strength - to an extravagantly high value, but that one must aim at a combination of several properties which make a polymeric material particularly valuable and attractive for a special use. For a rubber tube, f.i., one will require softness, flexibility, elasticity, abrasion resistance, impermeability to air and resistance against temperatures up to about $150^{\circ} \mathrm{C}$.

For the use as structural units in the construction industry one may characterize a favorable compromise of properties as follows:

(a) High modulus of rigidity; if possible higher than 700,000 psi.

(b) High softening or melting point; if possible higher than $500^{\circ} \mathrm{C}$.

(c) High tensile strength; if possible higher than 100,000 psi.

(d) High elongation to break; if possible above $10 \%$. Favorable values of (c) and (d) give. a large area under the stress-strain curve, which means a large requirement of energy to break or tear a piece of the material. This, in turn, manifests itself as a high impact strength and a high abrasion resistance.

「高分子」 
(e) High resistance against the action of solvents and swelling agents even at elevated temperature.

(f) High resistance against deterioration through heat, radiation and agressive chemical reagents.

Every material with the low specific gravity and low cost of organic polymers will find numerous profitable applications if one succeeds to incorporate into it a somewhat favorable combination of the above enumerated properties.

\section{Crystallization and Crosslinking}

It might therefore be useful to recapitulate very briefly the different principles with the aid of which one has been already able to achieve reasonably well balanced compromises of these properties and to explore whether and how one possibly could arrive at still better combinations. In a general anh simplified sense one can say that there are mainly three principles which have been useful in the past and should be good working hypotheses for future efforts:

(a) Crystallization

(b) Crosslinking, and

(c) The use of inflexible chain molecules.

Let us, therefore, estimate the value of each of these principles for the present purpose and, at tde end, consider how combinations of them could be used to arrive at improved products.

The capacity to crystallize has long been known to be a very valuable property of linear, flexible macromolecules whenever one wants a good combination of thermal and mechanical properties. Even linear polyethylene, a completely non polar material with weak interchain bonding is rigid, relatively high melting $\left(130^{\circ} \mathrm{C}\right)$, strong, tough, abrasion resistant and insoluble in anything at room temperature only because it possesses a strong tendency to crystallize, which means in this context, to form lamellae, bundles or domains of high lateral order. The same thing is true for isotactic polypropylene with a melting point of $170^{\circ} \mathrm{C}$ and for isotactic polystyrene, which melts around $230^{\circ} \mathrm{C}$. If the macromolecules contain polar groups and are of regular architecture even better combinations of mechanical and thermal properties have been materialized such as in polyvinyl alcohol, polyvinylidene chloride, polyoxyme- thylene and many aliphatic polyesters and polyamides like polycaprolactam or 66 and 610 nylon.

In all these cases we have intrinsically flexible chain molecules of regular architecture which have a distinct tendency to form crystalline domains inside of which a systematic accumulation of the interchain forces rigidifies and reinforces the entire structure to such an extent that the system becomes hard, high softening and difficultly soluble.

It has also long been known that a combination of the favorable properties enumerated on page 13 can be obtained in an entirely different manner, namely by the chemical crosslinking of long flexible chain molecules. Rubber is a typical example. As one reduces the original segmental mobility of the individual chains by the establishment of localized but strong carbon-surfur and sulfur-sulfur crosslinks the material becomes more rigid, higher softening and less soluble. If one continues to introduce more and more corsslinks, their average distances along the flexible chains decrease, the system is progressively stiffened and one finally winds up with hard rubber or Ebonite, a material which is rigid, has a high softening range and is completely insoluble and unswellable.

The effect of crosslinking depends on a number of factors:

(a) The length of the crosslinking element is of importance because very short crosslinks such as direct bonds between the carbon atoms of two chains, methylene $\left(-\mathrm{CH}_{2}-\right)$ bridges or -S-, -S-S-' - S - bonds create stiffness and high softening sooner II

than longer crosslinks such as those provided by the use of diamines, diepoxides, diolefins, dialdehydes and diisocyanates. Apparently the relatively flexible chains of such crosslinks permit to maintain segmental mobility even if the tiepoints are relatively. close together and bulid up three-dimensional networks of remarkable resilience, toughness and recovery power.

(b) The chemical nature of the crosslinks is important for their resistance to elevated temperatures and to chemical reagents. Thus -C-C- crosslinks are very resistant against both influences, sulfide $\left(-\mathrm{S}^{-}\right)$, disulfide $(-\mathrm{S}-\mathrm{S}-)$ and ester $\left(-\mathrm{CO}^{-} \mathrm{O}^{-}\right)$ crosslinks are sensitive against heat and alcali, acetalic $\left(-\mathrm{O}-\mathrm{CH}_{2}-\mathrm{O}-\right)$ tie points are sensitive to 
acids and urethane type (-NH-CO-O-) bonds are cleaved at elevated temperatures. Depending upon the nature of the base polymer there is a rather wide choice of crosslinking agents available which permit to produce threedimensional networks with desired properties.

(c) It should be added here that an effect similar to that of crosslinking can also be obtained by the incorporation of a reinforcing filler into a polymer. The word reinforcing refers to the fact there is a very intimate contact between the filler and the chains of the polymer in molecular dimensions and that there exist strong adsorption forces which fix and immobilize the polymer chains at the surface of the filler particles.

Although, at the first glance, the effect of crosslinking appears to be very similar to that of crystallization, there are several important defferences:

(a) In a crystalline system the rigidity is tie result of many, regularly spaced lateral bonds between the oriented chains; each of these bonds is weak and the ultimate effect comes from their large number and their regularity. In a crosslinked system the bonds between the long flexible chains are strictly locarized, each of them is strong; in their entirety they are randomly arranged in the system. As a result, crystallization is a reversible phenomenon, whereas crosslinking is irreversible and is the preferred technique for the production of thermosetting resins.

(b) Crystallization is a physical effect which takes place at all temperatures and is strongly influenced by mechanical processes such as orientation and relaxation; crosslinking, on the other hand, is a chemical phenomenon which needs the presence of certain special reagents, is strongly accelerated by elevated temperatures, but not very much influenced by orientation or relaxation.

Many important products which are hard, infusible and insolble are made with the aid of crosslinking such as all hard rubbers, urea-, melamine and phenol formaldehyde, condensation products, polyesters which use glycerol, trimethylol propane or pentaerythritol as components and resins which are hardened by the grafting of styrene on a polyester backbone which contains aliphatic double bonds. While there are obvious and significant differences in the way in which crystallization viz. crosslinking act, it was very welcome to have two different principles to attain a desirable combination of valuable properties. Even more encouraging is the fact that there exists a third independent principle to arrive at the same result, namely the use of 'stiff linear chain molecules.

\section{Inflexible Chain Molecules}

Crystallization and crosslinking produce stiffness, high softening and difficult solubility by the establishment of firm lateral connections between intrinsically flexible chains; one can, however, also obtain the same result by incorporating the stiffness in the individual chains and constructing them in such a manner that their segmental motion is intrins!cally restricted. One step in this direction is the introduction of bulky substituents into chains with flexible backbones. Polystyrene for instance is amorphous, has no crosslinks, but still is a hard, relatively high softening $\left(90^{\circ} \mathrm{C}\right)$ polymer. The absence of crystallinity results in complete transparency, the absence of crosslinking in reversible moldability and easy flow characteristics. Similar conditions prevail in the case of polymethyl mechacrylate which is linear, amorphous, has intrinsically flexible backbone chains which, however, are stiffened by the two substituents $\left(\mathrm{CH}_{3}\right.$ and $\left.\mathrm{COOCH}_{3}\right)$ at every other carbon atom and, as a result, is a hard, brilliantly transparent, relatively high softening (95 $\left.{ }^{\circ} \mathrm{C}\right)$ thermoplastic polymer, which has found many useful and valuable applications of both materials is their low resistance against swelling and dissolution; it seems that the bulky and eventually polar substituents are capable to produnce favorable mechanical and thermal effects which are connected with the overall mobility and flexibility of the chain segments but cannot offer sufficicnt resistance to the penetration of the system by solvents or swelling agents, because this process is a strictly localized phenomenon and depends on the affinity of the substituents for the particular solvent molecules.

Similar effects are also produced if the backbone chains themselves are rigid and if their substituents are so arranged that crystallization is prevented. Classical examples for such polymers are cellulose acetate and cellulose nitrate, where the glucosidic backbone chains possess a considerable intrinsic 
stiffness and the irregularly arranged acetyl-and nitrate groups of incompletely substituted specimens do not permit the formation of a crystalline order. In fact both materials are hard, transparent, high melting and amorphous thermoplastic resins which are widely and successfully applied since many years; cellulose acetate is still rather sensitive to the action of solvents and swelling agents just like polystyrene and polymethacrylate whereas collulose nitrate only dissolves in a few selected systems.

Recently the principle of using intrinsically rigid chains has been studied in more detail and has found several new and interesting embodiments Based on rigid monomeric units, such as

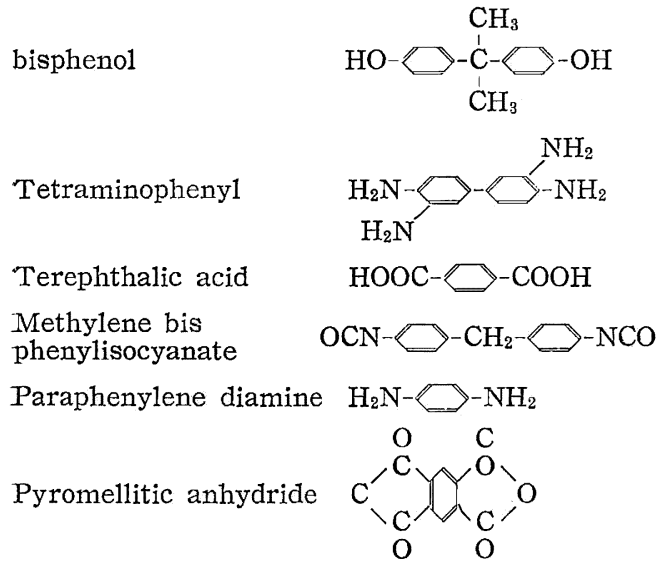

and others, a series of polymers have been synthesized which are substantially amorphous and uncrosslinked but represent very hard, high softening and solvent resistant materials. Earlier examples for this type are the polycarbonates and the linear epoxy resins which are both based on bisphenol; more recent representatives are polyphenylene oxides, polyphenylene sulfides, polyoxylylenes, polybenzimidazoles, polyimides, polyphenyl oxazoles and others, all of which exhibit unusually high resistance against softening, swelling and decomposition. In fact, some of these newer materials can stand temperatures up to $500^{\circ} \mathrm{C}$ for long periods without softening and deterioration and are completely insoluble in all organic solvents up to $300^{\circ} \mathrm{C}$.

Other rigid molecules which are now being studied for possible application in the field of high temperature resistant materials are based on other aromatic chains such as polyphenylene

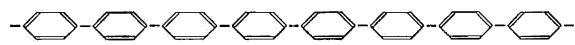

which cannot fold even at rather high temperatures

Vol. 16, No. 182 because rotation about the carbon-carbon single bond between the para-combined phenylene rings can only lead to different angles between the planes of consecutive rings but not to a kink or bend in the main chain. In fact, representatives of this species are rigid, high melting, possess a pronounced tendency to crystallize and are highly insoluble.

This combination of valuable properties of poly-para-phenylenes is now intensely investigated and several types with relatively high molecular weight have already been synthesized.

Para-polyphenylene oxide and polyphenylene sulfide are other cases which show the chain stiffening action of a para-phenylene unit.

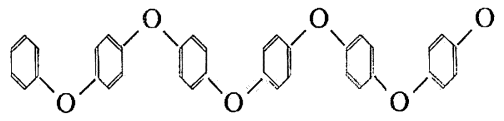

Rotation about the bonds between an ether oxygen atom and the adjacent carbon atoms of the rings does change the plane of the angle between two units and thus leads to bends and kinks in the chain, but the rotational freedom is noticeably inhibited by the presence of the aromatic rings on each side of the oxygen or sulfur atom. As a consequence, it has been found that chains of this type represent high melting, rigid and relatively insoluble materials.

Another interesting way to arrive at chains made up of condenced rings is the synthesis of socalled ladder polymers. The first case of such a structure was prepared by exposing polyacrylonitrile to elevated temperatures, which causes the formation of rows of fused six membered rings by an electron pair displacement:

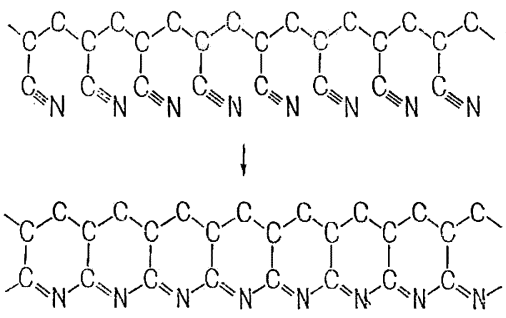

which involves stiffening, insolubility and discoloration. Further heating leads to evolution of $\mathrm{H}_{2}$ and to aromatization.

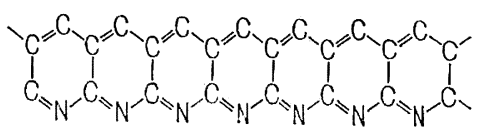


whereby a black, completely infusible and insoluble material is obtained which, in its structure corresponds to a linear graphite in which one carbon atom of every ring has been replaced by nitrogen.

These examples show that there are many possibilities for the formation of long stiff chains and that, in all cases, the properties of the resulting materials confirm the expectations.

The existence of three different and independent ways to establish favorable compromises of valuable properties stimulates the attempt to explore combinations of these principles and to see whether they might lead to even better rusults. To get a convenient survey of such combinations, let us consider a triangle (figure 1) in which the three principles of crystallization (A), crosslinking (B), and chain stiffening (C) are represented by the three corners $\mathrm{A}, \mathrm{B}$ and $\mathrm{C}$.

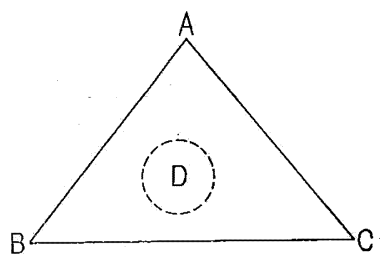

A : represents the principle of crystallization $B$ : represents the principle of crosslinking

$C:$ represents the principle of chain stiffening

Fig. 1 Illustration of three principles which influence polymer proporties

The corner A is populated by a large number of crystallizable, thermoplastic polymers with flexible chains which have proved to be particularly successful as fiber and film formers, Representative materials are polyethylene, polypropylene, polyoxymethylene, polyvinylalcohol, polyvinylchloride, polyvinylidene chloride and such polyamides as 6 and 66 nylon.

In the corner B are located the typical thermoset, highly crosslinked systems such as hard rubbers, urea-, melamine-, and phenolformaldehyde condensates, highly reticulated polyesters, polyepoxides, and polyurethanes.

Finally corner $\mathrm{C}$ is representative for the amorphous, thermoplastic resins with relatively high rigidity and high softening range such as polystyrene, polymethylmechacrylate, ABS resins, polystyrene derivatives and more recently polycarbonates, linear polyepoxides, polyethers and polycondensation pro- ducts with inflexible chains.

\section{Combined use of all three principles}

One can now pose the question, whether a combination of two principles has helped to arrive at products with more attractive and valuable properties and is, hereby, led to the exploration of the sides of the triangle of figure 1 .

In fact the line from $A$ to $C$ accommodates several interesting fiber and film formers. One of them is polyethylene glycol terephthalate (Terylene or Dacron) in which the para-phenylenic units of the acid introduces enough chain stiffening to bring the melting point of this polymer up to about $260^{\circ} \mathrm{C}$ which is as high as the melting point of 66 nylon although a polyester has no lateral hydrogen bonding available for the stiffening of its solid crystalline phase. Thus chain stiffening cooperates with crystallization to produce attractive properties without bringing either of these two principles to an extremely high value. Cellulose is another case $i_{n}$ which excellent fiber and film forming properties are built up by a combination of chain stiffening and crystallinity; these two effects together produce a polymer which is extremely rigid, does not melt at all and is soluble only in a very small number of particularly potent liquid systems. The presence of substantially rigid chains has the favorable consequence that high tensile strength and high softening characteristics become already apparent at relatively low degrees of crystallinity which places celluose somewnere in the middle of the line connecting $\mathrm{A}$ and C. Many of the recent spectacular improvements of cellulosic filaments are founded on a capitalization of the origin of its fiber forming potential. Another example for a beneficial combination of $\mathrm{A}$ and $\mathrm{C}$ is given by cellulose triacetate in which the capacity to crystallize superimposes several favorable properties on the normal cellulose acetate particularly insolubility in many organic liquids and heat settibillity through additional crystallization.

On the side $A-B$ are situated all those rubbers which are slightly or moderately crosslinked and crystallize on stretching progressivery; examples are natural rubber, high cis polybutadiene and polyisoprene, Butyl rubber and Neoprene. Depending upon the degree of crosslinking, they are more or less close to $\mathrm{B}$. 
Until now we have only considered systems of one component, namely a specific polymer or copolymer; in the technology of elastomers it is, however, customary to produce stiffening and temperature resistance by the addition of a hard, finely divided solid filler such as carbon black, silica or alumina. This addition of a crystalline or pseudocrystalline reinforcing filler is a kind of crystallization of the system in the sense that the flexible chains of the original polymeric matrix are restricted in the segmental mobility by the presence of the very small and hard particles of the filler to the surface of which they are attached by strong adsorptive forces. Thus the presence of a reinforcing filler simulates and replaces crystallization and brings the system closer to point $A$.

The line from $\mathrm{C}$ to $\mathrm{B}$ has been populated with certain useful polymers in the attempt to increase rigidity, high softening and insolubility of stiff chain systems by additional crosslinking. Well known cases for the combination of these two principles are the raising of the heat distortion point of acrylicand methacrylic polymers by the incorporation of allylmethacrylate or ethyleneglycol dimethacrylate and the "curing" of epoxy polymers which are based on such stiff chain elements as bisphenol and cyclic acetals of pentaerythritol. Additional attempts are now under way to improve the properties of the more recent amorphous systems with intrinsically stiff chains by cautious crosslinking and to move with these materials also from point $\mathrm{C}$ somewhat in the direction of point. The principal reason for this approach is to improve for such systems the resistance against dissolution and swelling at elevated temperatures.

The advantageous combination of two principles poses the question whether or not a proper combination of all three of them could lead to stillfurther improvements in property compromises. This is a field in which, at present, much exploratory work is done and in which already certain interesting results have been obtained. One successful application of all three principles is the aftertreatment of cotton with certain crosslinking agent or the spinning of rayon in the presence of such agents. Cellulose has rigid chains which can be brought to a moderate degree by the swelling of cotton or the appropriate spinning of rayon; the result are fibers of satisfactory strength, elongation, hand and dyeing characteristics but of insufficient recovery power. The introduction of a cautiously controlled system of crosslinks with the aid of bifunctional reagents leaves all other desirable properties unchanged and improves substantially the recovery power and wrinkle resistance. Similarly promising results have been obtained with mildly reticulated amorphous stiff, chain systems of the epoxy and urethane type in which crystallization has been replaced by a reinforcing filler.

The combinations of all three principles are situated somewhere in the interior of the triangle around $\mathrm{D}$ and one expect that thorough and systematic exploration of this area will lead to many new and interesting polymeric systems with properties superior to those which are at our disposal today.

\section{勤務先·住所などの変更についてのお願い}

最近，会誌・論交集などの刊行物の返送されてくるるのがとみに多くなって颉ります。住所・勤務先などの移

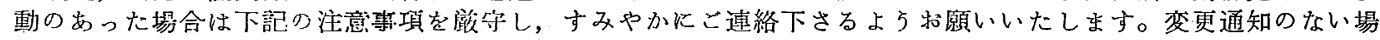
合，当学会よりの諸䢎知や䇣内，会誌その他の刊行物などがとどきまやんし，近々刊行予定で目下整備中の名簿

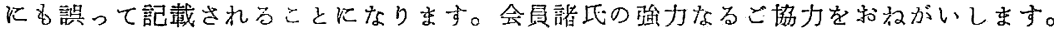

移動通知は下記のひな型に㳠い，官製ハオ゙キを使用することを必ず括守り下さい。

\begin{tabular}{|c|c|}
\hline 会貝 No. & 氏 名 \\
\hline 惧新先(听在地)（新) & (旧) \\
\hline 住所(自宅)（新） & （旧） \\
\hline 連絡先（新） & ( $\mid$ F|) \\
\hline
\end{tabular}

Dむて先 高分子学会会員課 東京都中央区銀座東 5 の 2 の 本州ビル 4 階 